\title{
Comparison of Gender, Age, and Body Mass Index Levels for Spatiotemporal Parameters of Bilateral Gait Pattern
}

\author{
Turki S. Abualait ${ }^{1}$ and Mohammad Ahsan ${ }^{1, *}$ \\ 1 Department of Physical Therapy, College of Applied Medical Sciences, Imam Abdulrahman Bin Faisal \\ University, Dammam, Saudi Arabia; tsabualait@iau.edu.sa; ORCID ID: 0000-0001-8534-6171 \\ * Correspondence: mahsan@iau.edu.sa; tel: +966 (0) 13333 31244; fax: +966 (0) 13333 0225; ORCID ID: 0000- \\ 0003-0232-3658
}

Running title: Spatiotemporal Analysis of Bilateral Gait

\begin{abstract}
Studies on the gait's parameters have been identified on the patients population. Most researchers confirm that the patients walk differently than normal people and they may have a risk for falls. Consistent finding and description of gender, age, and body mass index differences in gait studies is rare in healthy subjects. A cross-sectional study with forty-five young adult $(F=20, M=$ 25) was conducted. Stadiometer and Physilog 4 inertial sensors were used for data collection. A gait analyser 5.2 software (GaitUp, S.A. Lausanne, Switzerland) was used to determine spatiotemporal parameters. No statistically significant differences were found in any bilateral foot gait parameters with respect to gender, age, and body mass index. Females are found with higher total double support and cadence than males. Cadence also increases with age. Obese people showed lower gait speed, cadence, and total double support. These findings may be beneficial to those who have abnormal gait pattern due to age, body mass index differences, decreased muscle strength, spasticity, and joint mobility. This important informations should be considered to rehabilitate patients with abnormal gait patterns to controlling dynamic balance and riks to falling.
\end{abstract}

Keywords: spatiotemporal parameters; gait; gender; age; Body Mass Index

\section{Introduction}

Spatiotemporal is the primary measurement in gait analysis. There is a common perception that males walk differently as females. The differences may occur due to physical capacities as muscular strength, endurance, coordination, flexibility, agility, and emotional balance. Walking patterns may also fluctuate by age, body mass index (BMI), surface, course of time, and it changes from stride to stride. Gender differences in a healthy population reveal contradictory discoveries regarding spatiotemporal parameters of gait. stride-to-stride variability has been attributed to the underlying mechanism that produces human gait in healthy people ${ }^{[1]}$.

Many studies have been surprisingly investigating limited, contradictory, or equivocal between genders. One study reported that stride characteristics do not have any gender differences during walking ${ }^{[2]}$. Another study revealed that during walking, the speed is the same in males and females, but that the step length is shorter in females [3]. On the other hand, a study reported males to walk faster than females, and female step length is shorter than males ${ }^{[4]}$. A previous study concluded that healthy older females walk with shorter stride length and higher cadence while comparing to males [5]. Female has been indicated to walk with lower preferred speed, smaller step length, but increased cadence compared with male ${ }^{[6]}$. It has been found that healthy females had a higher velocity and with bigger cadence, swing phase, stride length, and single support phase and a lower double support phase and stance phase compared with gait disorder [7]. Heredia-Jimenez and OrantesGonzalez reported while dealing with healthy females and females with fibromyalgia there were significant differences in stride length, velocity, swing time variability, cadence, and stance gait ${ }^{[8]}$. 
Several pieces of research also concluded that mean stride times to be affected by gender, whereas other temporal parameters such as stance, swing, double support times, and temporal variability unchanged in healthy young adults and older peoples ${ }^{[9,2]}$.

Advance aging factors affect gait pattern. Many studies have been reported that gait ability decline with age. A prior study found that age differences are connected with slower gait speed, shorter stride length, and wider stride width ${ }^{[10]}$. Compare to young adults, the elderly adult gait model is characterized by shorter step length, slow gait speed, reduce the range of motion at the hip [11]. A study showed that when walking on a compliant surface, young and older peoples increase cadence and reduce velocity ${ }^{[12]}$. One of the most consistent age-related changes has been shown as a decline in gait speed ${ }^{[13]}$. There are many fall injuries associated with increasing age, from young adult to middle-aged adults to older adults. The aging process is accompanied by changes in body composition [14].

Obesity is a primary risk factor for many diseases and also negatively affects physical functioning, especially walking ability and performance [15]. The effects of obesity and overweight on gait parameters in adults not much known. In obese adults, gait is distinguished by slow step frequency, shorter step length, longer stance phase, and walking speed is minimized [14]. Stride frequency and stride length did not differ between moderately obese individuals and normal-weight individuals [16]. Obese individuals walk slowly with a shorter step length than underweight individuals [17]. There is a lack of knowledge on spatiotemporal parameters from the underweight, healthy, overweight, and obese population.

Many methods were suggested to investigate the spatiotemporal patterns of the gait sequence to understand the differences between males and females. Thang et al. used only the smartphone's accelerometer sensor to user authentication while Zong and Deng used to capture walking information from both the accelerometer and gyroscope sensors $[18,19]$. Recently, the Physiolog gait analysis system from GaitUp (SA, Lausanne, Switzerland) has become a popular and important tool for the objective evaluation and planning of rehabilitation strategies for an abnormal gait pattern. The use of these sensors has been described previously, and they demonstrate good accuracy and perception of gait analysis [20, 21]. Despite this evidence, a limited number of studies have investigated the spatiotemporal parameters of gait using internal wearable sensors.

Therefore, the purpose of this current study was to compare the characteristics of spatiotemporal gait based on gender, age, and BMI level. In particular, the aim of the study is to answer the question of whether there are differences between gait parameters. To achieve the objective of the study for gait parameters in gender, age, and BMI level, we tried a statistical comparison between male and female, age differences, and BMI categories for spatiotemporal gait analysis.

\section{Methodology}

Study Design

Cross-sectional study design was chosen to achieve the objective of this study. This study was conducted in accordance with the Declaration of the Principles of Helsinki. The Deanship of Scientific Research at Imam Abdulrahman bin Faisal University approved the study protocol. Informed consent was obtained from all participants.

\section{Sample Size}

The sample size was calculated by using (http://www.sample-size.net/correlation-sample-size/) web page. Data from (Lewek et al., 2014b) was used to calculate the sample size ${ }^{[22]}$. The total number of participants was calculated as 43 subjects.

Total sample size $=45$

The standard normal deviate for $\alpha=Z \alpha=1.960$

The standard normal deviate for $\beta=\mathrm{Z} \beta=.842$

$\mathrm{C}=0.5 \mathrm{x}$ in $[(1+\mathrm{r}) /(1-\mathrm{r})]=.375$ 


\section{Participants}

Forty-five young adults (twenty-five male and twenty female) were included in this study. Their mean (standard deviation [SD]) age, height, weight, and BMI were 21.82(3.93) years, 165.83(8.00) cm, $66.10(13.19) \mathrm{kg}$, and 24(3.89) respectively. All participants free from injury, disease, or musculoskeletal disorder, which could alter their walking performance. All the participants were flat shoes during the test.

Table 1. Anthropometric measurement of participants.

\begin{tabular}{ccccc}
\hline & $\begin{array}{c}\text { Male } \\
\text { Mean } \pm \text { SD }\end{array}$ & $\begin{array}{c}\text { Female } \\
\text { Mean } \pm \text { SD }\end{array}$ & $\begin{array}{c}\text { Both } \\
\text { Mean } \pm \text { SD }\end{array}$ & Sig. \\
\hline Age (Years) & $24.88 \pm 5.60$ & $23.75 \pm 6.41$ & $24.72 \pm 6.43$ & .314 \\
\hline Height (cm.) & $170.78 \pm 6.25$ & $159.65 \pm 5.15$ & $165.83 \pm 8.00$ & .000 \\
\hline Weight (kg.) & $71.90 \pm 12.40$ & $58.85 \pm 10.45$ & $66.10 \pm 13.19$ & .001 \\
\hline Leg length (cm.) & $99.7 \pm 2.6$ & $91.6 \pm 2.7$ & $95.65 \pm 2.65$ & .084 \\
\hline BMI & $25.53 \pm 3.92$ & $22.80 \pm 3.37$ & $24.32 \pm 3.89$ & .018 \\
\hline
\end{tabular}

\section{Equipment}

Stadiometer cum weighing scale

Weight, height, and BMI were measured with a portable electronic calibrated scale (Detecto Scale-model 750, USA). Participants were asked to wear light clothing and take off their shoes for accurate measurement.

\section{Physiolog 4}

To measure spatiotemporal parameters of all participants Physilog 4 silver 10D from GaitUp (S.A., Lausanne, Switzerland) was used. Physilog has good accuracy and precision for gait analyses [20, 21].

\section{Procedure}

A total of forty-five participants agreed to participate in the study. There were 20 female and 25 male participants. Before the actual test, all basic instructions are explained to the participants. After collecting their anthropometric data with a Detecto scale, physilog inertial sensors were placed on each participant's foot for the gait test. Participants were asked to walk at a comfortable pace of themselves, along a 10-meter straight path. The three trails were recorded. The average of the three trials was used for further analysis. In this study, the spatiotemporal parameters chosen for the analysis were gait speed, gait cycle, foot speed, stride length, total double support, and cadence for both legs. A gait analyser 5.2 software (GaitUp, S.A. Lausanne, Switzerland) was used to determine stride length, stride speed, standing interval, and stride length.

\section{Statistical Analysis}

Statistical analysis was performed using IBM SPSS for windows, version-21 (IBM Crop. USA). The data has usually been calculated. Descriptive analyzes were conducted for anthropometric characteristics. Independent sample $t$-tests and analysis of the variance test was extended to find out the differences between different types of spatiotemporal parameters of gait for gender, age, and BMI levels. The significance level was set at the 0.05 level.

\section{Results}

Table 2. A comparative analysis between male and female participants.

$\begin{array}{lllll}\text { Gait Parameters } & \text { Males } & \text { Females } & \text { Std. Error Difference } & \text { Sig. }\end{array}$




\begin{tabular}{ccccc}
\hline & Mean \pm SD & Mean \pm SD & & 0.033 \\
\hline Gait Speed Left & $1.18 \pm 0.10$ & $1.16 \pm 0.12$ & 0.036 & 0.631 \\
Gait Speed Right & $1.17 \pm 0.12$ & $1.13 \pm 0.12$ & 0.029 & 0.360 \\
Gait Cycle Left & $1.14 \pm 0.11$ & $1.12 \pm 0.08$ & 0.030 & 0.358 \\
Gait Cycle Right & $1.15 \pm 0.10$ & $1.15 \pm 0.10$ & 0.103 & 0.926 \\
Foot Speed Left & $3.71 \pm 0.37$ & $3.62 \pm 0.32$ & 0.126 & 0.373 \\
Foot Speed Right & $3.64 \pm 0.49$ & $3.62 \pm 0.32$ & 0.024 & 0.904 \\
Stride Length Left & $1.29 \pm 0.09$ & $1.28 \pm 0.06$ & 0.025 & 0.764 \\
Stride Length Right & $1.29 \pm 0.09$ & $1.25 \pm 0.06$ & 1.121 & 0.176 \\
Total Double Support Left & $20.02 \pm 3.81$ & $21.67 \pm 3.65$ & 1.122 & 0.147 \\
Total Double Support Right & $19.99 \pm 3.81$ & $21.67 \pm 3.65$ & 2.210 & 0.140 \\
Cadence Left & $108.13 \pm 6.85$ & $108.60 \pm 7.97$ & 2.045 & 0.834 \\
Cadence Right & $108.83 \pm 6.07$ & $108.88 \pm 7.66$ & 0.981 \\
\hline
\end{tabular}

The results of the gait parameters for males and females' scores are presented in table-2. When considering the mean score, males have higher gait speed, gait cycle, foot speed, and stride length than females, while females have higher total double support and cadence than males. Independent sample t-tests were conducted to compare the left and right foot's gait speed, gait cycle, foot speed, stride length, total double support, and cadence for the males and females. There were no significant differences found in any gait parameters in the left and right foot for males and females participants.

Table 3. A comparative analysis for age categories.

\begin{tabular}{ccccc}
\hline Gait Parameters & $\begin{array}{c}\text { Age (18-25) } \\
\text { Mean } \pm \text { SD }\end{array}$ & $\begin{array}{c}\text { Age (30-38) } \\
\text { Mean } \pm \text { SD }\end{array}$ & Std. Error Difference & Sig. \\
\hline Gait Speed Left & $1.18 \pm 0.12$ & $1.15 \pm 0.07$ & 0.036 & .413 \\
Gait Speed Right & $1.15 \pm 0.14$ & $1.15 \pm 0.08$ & 0.039 & .831 \\
Gait Cycle Left & $1.13 . \pm 0.10$ & $1.13 \pm 0.08$ & 0.031 & .918 \\
Gait Cycle Right & $1.14 \pm 0.11$ & $1.15 \pm 0.09$ & 0.032 & .849 \\
Foot Speed Left & $3.70 \pm 0.38$ & $3.61 \pm 0.24$ & 0.111 & .454 \\
Foot Speed Right & $3.68 \pm 0.38$ & $3.51 \pm 0.48$ & 0.132 & .203 \\
Stride Length Left & $1.30 . \pm 0.09$ & $1.26 . \pm 0.04$ & 0.025 & .182 \\
Stride Length Right & $1.28 . \pm 0.10$ & $1.26 . \pm 0.04$ & 0.027 & .450 \\
Total Double Support Left & $20.93 \pm 3.65$ & $20.37 \pm 4.19$ & 1.230 & .652 \\
Total Double Support Right & $20.90 \pm 3.66$ & $20.37 \pm 4.19$ & 1.233 & .666 \\
Cadence Left & $108.27 \pm 7.97$ & $108.48 \pm 5.76$ & 2.373 & .931 \\
Cadence Right & $108.77 \pm 7.42$ & $109.03 \pm 6.74$ & 2.194 & .906 \\
\hline
\end{tabular}

The result of gait parameters based on age categories (18-25 and 30-38 years) scores are presented in table-3. When considering the mean scores, the participants belong to the 18-25 years age category showed higher gait speed, gait cycle, foot speed, stride length, and total double support than participants belong to the 30-38 years age category. While the participants belong to the 30-38 years age categories showed higher left and right foot's cadence than the participants belong to the 18-25 years age category. Independent sample t-tests were conducted to compare the left and right foot's gait speed, gait cycle, foot speed, stride length, total double support, and cadence for the age categories. The results showed that there were no significant differences found among any gait parameters in left and right foot for any age categories i.e. 18-25 and 30-38 years.

Table 4. A comparative analysis between Body mass index level.

\begin{tabular}{cccccc}
\hline Gait Parameters & $\begin{array}{c}\text { Underweight } \\
\text { Mean } \pm \text { SD }\end{array}$ & $\begin{array}{c}\text { Healthy } \\
\text { Mean } \pm \text { SD }\end{array}$ & $\begin{array}{c}\text { Overweight } \\
\text { Mean } \pm \text { SD }\end{array}$ & $\begin{array}{c}\text { Obese } \\
\text { Mean } \pm \text { SD }\end{array}$ & Sig. \\
\hline Gait Speed Left & $1.26 \pm 0.12$ & $1.18 \pm 0.10$ & $1.15 \pm 0.13$ & $1.11 \pm 0.11$ & .371 \\
Gait Speed Right & $1.19 \pm 0.08$ & $1.17 \pm 0.10$ & $1.11 \pm 0.16$ & $1.13 \pm 0.17$ & .558 \\
Gait Cycle Left & $1.04 \pm 0.06$ & $1.13 \pm 0.08$ & $1.15 \pm 0.12$ & $1.17 \pm 0.16$ & .471 \\
Gait Cycle Right & $1.14 \pm 0.09$ & $1.13 \pm 0.09$ & $1.17 \pm 0.10$ & $1.17 \pm 0.17$ & .737 \\
Foot Speed Left & $3.84 \pm 0.14$ & $3.70 \pm 0.35$ & $3.52 \pm 0.36$ & $3.82 \pm 0.24$ & .314 \\
Foot Speed Right & $3.78 \pm 0.33$ & $3.62 \pm 0.47$ & $3.57 \pm 0.36$ & $3.78 \pm 0.23$ & .821
\end{tabular}


Stride Length Left

Stride Length Right

Total Double Support Left

Total Double Support Right

Cadence Left

Cadence Right
$1.31 \pm 0.05$
$1.24 \pm 0.03$
$16.03 \pm 0.17$
$16.03 \pm 0.17$
115.566 .60
$116.04 \pm 5.56$

$1.29 \pm 0.09$

$1.29 \pm 0.07$

$20.82 \pm 3.50$

$1.26 \pm 0.08$

$1.24 \pm 0.11$

$20.79 \pm 3.15$

$21.99 \pm 3.53$

$21.99 \pm 3.53$

$108.64 \pm 6.08$

$107.17 \pm 8.08$

$109.10 \pm 5.99$
$1.29 \pm 0.06$

$1.26 \pm 0.07$

$19.24 \pm 5.94$

$19.24 \pm 5.94$

$105.83 \pm 12.86$

$107.80 \pm 10.51$
.664

.444

.178

.179

.441

.405

The result of gait parameters on the basis of their BMI levels (underweight, healthy, overweight, and obese) scores are presented in table- 4 . When considering the mean scores, the participants belong to their BMI level showed that underweight participants have the highest gait speed, foot speed, stride length, and cadence than the participants belong to other BMI categories. While gait cycle and total double support for the left and the right foot are smallest than the other BMI categories participants. The participants belong to a healthy category show that the gait speed, foot speed, stride length, and cadence are higher and the gait cycle is smaller than overweight and obese participants. while total double support for the left and the right foot is lower than overweight and higher than obese participants. The overweight participants showed the gait speed, gait cycle, foot speed, the stride length is lower than obese participants. while total double support and cadence for left and right showed higher overweight than obese participants. The obese participants showed gait speed and cadence are lower and the gait cycle is higher than other BMI categories participants, foot speed is higher than health and overweight participants while lower than underweight participants. The total double support is lower than healthy and overweight but higher than underweight participants. ANOVA one-way was conducted to compare the gait parameters among different BMI categories participants. The results showed that there were no significant differences in any gait parameters for the left and right foot for any BMI categories' participants.

\section{Discussion}

The finding of this study indicated that gait speed, gait cycle, foot speed, and stride length was higher in male than female participants. Whereas cadence and double support were higher in female than male participants. These results suggest that there was no significant difference in male and female participants for the left and right foot gait parameters. Most previous studies suggested that step length and cadence are responsible for the gait speed, and these measurements have some biological dependence on the height of the individual [23]. Kerrigan et al. and colleagues have observed that healthy males who walked at the same walking speed as females showed lower cadence and longer step length than the female ${ }^{[24]}$. It was reported that healthy women walk with reduced stride length and higher cadence with respect to men, in order to achieve comparable speed values [5]. It was reported that spatiotemporal gait parameters for both genders showed that females have only greater stride time while males performed higher stride length, step time, cadence, and walking speed ${ }^{[25]}$. Another study revealed that there are few significant gender differences for spatiotemporal data as a longer normalized stride length and greater cadence in females. Both genders had the same step width and walking velocity due to the female efforts made to increase their stride length with the aim of the walk as fast as males ${ }^{[24]}$. We found that males and females did not differ significantly in the spatiotemporal parameters of normal gait speed, gait cycle, normal stride length, and cadence. These findings are in partial agreement with the findings of the above mentioned studies. Gender differences may also be associated with body proportions between males and females. Muscle strength and bony configuration may have importance to determine gait parameters outcomes between genders.

The result of gait parameters based on age categories (18-25 and 30-38 years) scores are presented in table-3. The participants belong to the 18-25 years age category showed higher gait speed, gait cycle, foot speed, stride length, and total double support than participants belong to the 22-28 years age category. While the participants belong to the 30-38 years age categories showed higher left and right foot's cadence than the participants belong to the 18-25 years age category. The results of the present study indicated that despite the similarities in terms of the main spatiotemporal parameters in the age group shown. Several studies indicated that these spatiotemporal measures deteriorate more rapidly with age for women than for men ${ }^{[26], ~ w h i l e ~ o t h e r s ~ f o u n d ~ n o ~ i n t e r a c t i o n s ~ b e t w e e n ~ t h e ~}$ 
sexes during aging [27]. Results from Frimenkoa et al. study indicated that there was a significant difference exists in both genders at slowed their gait speed with age. At a similar age, females have a higher cadence and smaller stride lengths than males. As the age increases gait speed decreases in both gender while, females maintain smaller step length and higher cadence [28]. Moreover, Abreu and colleagues uncovered a negative relationship between aging and stride length during gait due to increased eccentric activity of the quadriceps muscles during the final stage of double support, or increased eccentric activity in the hamstrings during the final balance phase that occurs with increasing age ${ }^{[29]}$. Older adults reduce their gait speed and take shorter steps while increasing the time of double support to maintain their dynamic balance [30]. The results of the present study also support previous findings that age-related changes in gait speed through shorter steps were adopted for a safer and more steady gait.

Our findings showed that the underweight participants have the highest gait speed, foot speed, stride length, and cadence than the participants belong to other BMI categories. While gait cycle and total double support for the left and the right foot are smallest than the other BMI categories participants. The participants belong to a healthy category show that the gait speed, foot speed, stride length, and cadence are higher and the gait cycle is smaller than overweight and obese participants. while total double support for the left and the right foot is lower than overweight and higher than obese participants. The overweight participants showed the gait speed, gait cycle, foot speed, the straight length is lower than obese participants. while total double support and cadence for left and right showed higher overweight than obese participants. The obese participants showed gait speed and cadence are lower and the gait cycle is higher than other BMI categories participants, foot speed is higher than health and overweight participants while lower than underweight participants. the total double support is lower than healthy and overweight but higher than underweight participants.

People who are overweight and/or obese are known to have a functional implication in everyday life. It has been shown that excess weight alters the normal gait mechanism ${ }^{[16]}$. Our findings are in line with previous research, with obese adults walking with shorter strides in length, large stride width, and shorter stride length compared to normal-weight adults when walking at a self-defined speed [31]. On the other hand, when comparing healthy and obese adults, no differences were found in step length ${ }^{[16]}$ and step length ${ }^{[17]}$. However, these results may be directly due to the effect of speed ${ }^{[16]}$. It has been clearly indicated in the literature that obese people tend to have reduced stride length, swing phase duration, cadence, walking speed, increased stance phase, step width, and double support [32]. A recent review summarizing the results of 25 studies on the gait of obese children ${ }^{[33]}$ concluded that there is moderate evidence of increased step width and stance phase duration, while for all other spatiotemporal parameters, the differences are either non-significant or inconsistent as our results suggest.

Limitations in this study included a small sample size of the healthy subjects from an institution and difficulty recruiting subjects secondary to the higher age and weight groups. Physilog (GaitUp) not a common means for determining spatiotemporal gait parameters, this may be employed more in future research. The results do not provide any cause and effect relationship.

\section{Conclusion}

In summary, we quantified the spatiotemporal parameters of gait differences as per gender, age, and BMI levels. Our result suggests that there are differences in all the spatiotemporal parameters for gender, age, and BMI levels at the left and right foot, but these differences are not statistically significant to each other. These findings may be beneficial to those who have abnormal gait pattern due to age, body mass index differences, decreased muscle strength, spasticity, and joint mobility. This important informations should be considered to rehabilitate patients with abnormal gait patterns to controlling dynamic balance and riks to falling.

\section{References}

1. Stergiou N, Moraiti C, Glakas G, Ristanis S, Georgoulis A. The effect of walking speed on the stability of the anterior cruciate ligament deficient knee, Clin Biomech, 19, 2004, 57-63. 
2. Barrett R, Noordegraaf MV, Morrison S: Gender difference in the variability of lower extremity kinematics during treadmill locomotion. J Mot Behav 2008, 40:62-70.

3. Laufer Y: Age and gender related changes in the temporal-spatial characteristics of forwards and backwards gaits. Physiother Res Int 2003, 8:131-142.

4. Samson MM, Crowe A, de Vreede PL, Dessens JA, Duursma SA, Verhaar HJ: Differences in gait parameters at a preferred waking speed in healthy subjects due to age, height and body weight. Aging 2001, 13:16-21.

5. Ko SU, Tolea MI, Hausdorff JM, Ferrucci L. Sex-specific differences in gait patterns of healthy older adults: results from the Baltimore Longitudinal Study of Aging. J Biomech 2011; 44:1974-1979.

6. Bohannon RW, Andrews AW. Normal walking speed: a descriptive meta-analysis. Physiotherapy 2011;97 (3):182-189

7. Heredia Jimenez JM, Aparicio Garcia-Molina VA, Porres Foulquie JM, Delgado Fernandez M, Soto Hermoso VM. Spatial-temporal parameters of gait in women with fibromyalgia. Clin Rheumatol (2009) 28:595-598.

8. Heredia-Jimenez J, Orantes-Gonzalez E. Gender differences in patients with fibromyalgia: a gait analysis. Clin Rheumatol, 2019 Feb;38(2):513-522.

9. Hollman JH, McDade EM, Petersen RC. Normative spatiotemporal gait parameters in older adults. Gait Posture 2011; 34:111-8.

10. Ko SU, Stenholm S, Ferrucci L. Characteristic Gait Patterns in Older Adults with Obesity - Results from the Baltimore Longitudinal Study of Aging, J Biomech. 2010; 43(6): 1104-1110.

11. Ko SU, Hausdorff JM, Ferrucci L. Age-associated differences in the gait pattern changes of older adults during fast-speed and fatigue conditions: results from the Baltimore longitudinal study of ageing. Age Ageing. 2010; 39:688-694.

12. Rogers HL, Cromwell RL, Grady JL. Adaptive changes in gait of older and younger adults as responses to challenges to dynamic balance. J Aging Phys Act. 2008;16(1):85-96.

13. Winter DA, Patla AE, Frank JS, Walt SE. Biomechanical walking pattern changes in the fit and healthy elderly, Phys Ther, 1990;70:340-347

14. Roubenoff R. Sarcopenia: Effects on Body Composition and Function. J Gerontol A BiolSci Med Sri 2003; 58:1012-1017.

15. Stenholm S, Sainio P, Rantanen T, Alanen E, Koskinen S. Effect of co-morbidity on the association of high body mass index with walking limitation among men and women aged 55 years and older. Aging Clin Exp Res. 2007;19(4):277-83.

16. Browning RC, Kram R. Effects of obesity on the biomechanics of walking at different speeds. Medicine and Science in Sports and Exercise, 2007;39(9):1632-1641.

17. DeVita $\mathrm{P}$, Hortobagyi T. Obesity is not associated with increased knee joint torque and power during level walking J Biomech. 2003;36(9):1355-1362

18. Thang HM, Viet VQ, Thuc ND, Choi D. Gait identification using accelerometer on mobile phone. International Conference on Control, Automation and Information Sciences (ICCAIS), 2012: 344-348.

19. Zhong Y, Deng Y. Sensor orientation invariant mobile gait biometrics. IEEE International Joint Conference on Biometrics (IJCB), 2014:1-8.

20. Bregou Bourgeois A, Mariani B, Aminian K, Zambelli PY, Newman CJ. Spatio-temporal gait analysis in children with cerebral palsy using, foot-worn inertial sensors. Gait Posture, 2014;39, 436-442.

21. Dadashi, F. et al. Gait and foot clearance parameters obtained using shoe-worn inertial sensors in a large-population sample of older adults. Sensors. 2013;14, 443-457.

22. Lewek MD, Bradley CE, Wutzke CJ, Zinder SM. 2014b. The relationship between spatiotemporal gait asymmetry and balance in individuals with chronic stroke. Journal of applied biomechanics, 30, 31-36.

23. Hof AL. Scaling gait data to body size. Gait Posture 1996;4(3):222-3.

24. Kerrigan DC, Todd MK, Croce UD. Gender differences in joint biomechanics during walking: normative study in young adults. Am. J. Phys. Med. Rehabil. 1998; 77, 2-7.

25. Zakaria NK, Jailani N, Tahir NM. Gender differences in gait features of healthy children. Jurnal Teknologi (Sciences \& Engineering), 2015;77(7):1-6 
26. Doyo W, Kozakai R, Kim HY, Ando F, Shimokata H. Spatiotemporalcomponents of the 3-D gait analysis of community-dwelling middle-aged and elderly Japanese: age-and sex-related differences. Geriatr Gerontol Int 2011;11(1):39-49.

27. Verlinden V, van der Geest J, Hoogendam YY, Hofman A, Breteler M,Ikram MA. Gait patterns in a community-dwelling population aged 50years and older. Gait Posture 2013;37(4):500-5.

28. Frimenkoa R, Goodyeara C, Brueningb D. Interactions of sex and aging on spatiotemporal metrics innon-pathological gait: A descriptive meta-analysis, Physiotherapy. 2015;101,266-272

29. Abreu SSE, Caldas CP. Gait speed, balance and age: A correlational study among elderly women with and without participation in a therapeutic exercise program. Revista Brasileira de Fisioterapia, 2008;12:324-330.

30. Prince F, Corriveau H, Winter DHR. Gait in the elderly. Gait Posture, 1997;5: 128-135.

31. Runhaar J, Koes BW, Clockaerts S, Bierma-Zeinstra SMA. A systematic review on changed biomechanics of lower extremities in obese individuals: A possible role in development of osteoarthritis. Obes. Rev. 2011; 12:1071-1082.

32. Wearing SC, Hennig EM, Byrne NM, Steele JR, Hills AP. The biomechanics of restricted movement in adult obesity. Obes. Rev. 2006; 7:13-24.

33. Molina-Garcia P, Migueles JH, Cadenas-Sanchez C, Esteban-Cornejo I, Mora-Gonzalez J, RodriguezAyllon M, Plaza-Florido A, Vanrenterghem J, Ortega FB. A systematic review on biomechanical characteristics of walking in children and adolescents with overweight/obesity: Possible implications for the development of musculoskeletal disorders.Obes Rev. 2019;20(7):1033-1044. 